\title{
A Modified Hermite Interpolation with Exponential Parameterization
}

\author{
R. Kozera · M. Wilkołazka
}

Received: 30 November 2017 / Revised: 14 May 2018 / Accepted: 24 May 2018 / Published online: 6 July 2018

(C) The Author(s) 2018

\begin{abstract}
This work discusses the problem of fitting a regular curve $\gamma$ based on reduced data points $Q_{m}=$ $\left(q_{0}, q_{1}, \ldots, q_{m}\right)$ in arbitrary Euclidean space. The corresponding interpolation knots $\mathcal{T}=\left(t_{0}, t_{1}, \ldots, t_{m}\right)$ are assumed to be unknown. In this paper the missing knots are estimated by $\mathcal{T}_{\lambda}=\left(\hat{t}_{0}, \hat{t}_{1}, \ldots, \hat{t}_{m}\right)$ in accordance with the so-called exponential parameterization (see Kvasov in Methods of shape-preserving spline approximation, World Scientific Publishing Company, Singapore, 2000) controlled by a single parameter $\lambda \in[0,1]$. In order to fit $\left(\hat{\mathcal{T}}_{\lambda}, Q_{m}\right.$ ), a modified Hermite interpolant $\hat{\gamma}^{H}$ (a $C^{1}$ piecewise-cubic) introduced in Kozera and Noakes (Fundam Inf 61(3-4):285-301, 2004) is used. The sharp quartic convergence order for estimating $\gamma \in C^{4}$ by $\hat{\gamma}^{H}$ is proved in Kozera (Stud Inf 25(4B-61):1-140, 2004) and Kozera and Noakes (2004) only for $\lambda=1$ and within the general class of admissible samplings. The main result of this paper extends the latter to the remaining cases of exponential parameterization covering all $\lambda \in[0,1)$. A slower linear convergence order in trajectory estimation is established for any $\lambda \in[0,1)$ and arbitrary more-or-less uniform sampling. The numerical tests conducted in Mathematica indicate the sharpness of the latter and confirm the necessity of more-or-less uniformity. Other interpolation schemes used to fit reduced data $Q_{m}$ and based on $\mathcal{T}_{\lambda}$ together with some relevant applications are also briefly recalled in this paper.
\end{abstract}

Keywords Interpolation $\cdot$ Reduced data $\cdot$ Convergence order $\cdot$ Numerical analysis

Mathematics Subject Classification 65D05 $65 \mathrm{D} 10 \cdot 65 \mathrm{D} 18$

This research was supported in part by PLGrid Infrastructure.

R. Kozera $(\bowtie)$

Faculty of Applied Informatics and Mathematics, Warsaw University of Life Sciences - SGGW, U1. Nowoursynowska 157,

02-776 Warsaw, Poland

e-mail: ryszard.kozera@gmail.com

R. Kozera

School of Physics, Mathematics and Computer Science, Computer Science and Software Engineering, The University of Western Australia, 35 Stirling Highway, Crawley, Perth, WA 6009, Australia

R. Kozera $\cdot$ M. Wilkołazka

Faculty of Mathematics, Informatics and Landscape Architecture, The John Paul II Catholic University of Lublin, Ul.

Konstantynów 1H, 20-708 Lublin, Poland

e-mail: magda.wilkolazka@gmail.com 


\section{Introduction}

Let $\gamma:[0, T] \rightarrow \mathbb{E}^{n}$ be a smooth regular curve (i.e. $\dot{\gamma}(t) \neq \overrightarrow{0}$ ) defined over $t \in[0, T]$, for $0<T<\infty$. The term reduced data $Q_{m}$ is defined by the sequence of $m+1$ interpolation points $\left\{q_{i}\right\}_{i=0}^{m}$ in arbitrary Euclidean space $\mathbb{E}^{n}$. In addition, we assume that for $q_{i}=\gamma\left(t_{i}\right)$ we have $q_{i+1} \neq q_{i}(i=0,1, \ldots, m-1)$. The respective knots $\mathcal{T}=\left\{t_{i}\right\}_{i=0}^{m}$ (admitted in ascending order) are here unavailable. Any fitting scheme $\hat{\gamma}$ interpolating reduced data $Q_{m}$ is called non-parametric interpolation. In order to derive an interpolant $\hat{\gamma}$, first the knot estimates $\left\{\hat{t}_{i}\right\}_{i=0}^{m} \approx\left\{t_{i}\right\}_{i=0}^{m}$ should be somehow guessed subject to the interpolation constraint $\hat{\gamma}\left(\hat{t}_{i}\right)=q_{i}$ and $\hat{t}_{i}<\hat{t}_{i+1}$. Upon selecting the specific scheme $\hat{\gamma}:[0, \hat{T}] \rightarrow \mathbb{E}^{n}$ fitting $Q_{m}$ and the associated substitutes $\hat{\mathcal{T}}=\left\{\hat{t}_{i}\right\}_{i=0}^{m}$ of the unknown knots $\left\{t_{i}\right\}_{i=0}^{m}$, the question concerning the intrinsic asymptotic order $\alpha$ in approximating $\gamma$ with $\hat{\gamma}$ arises naturally. In doing so, recall now some background notions (see e.g. [1,20]).

Definition 1.1 We say that sampling $\left\{t_{i}\right\}_{i=0}^{m}$ is admissible if:

$\lim _{m \rightarrow \infty} \delta_{m}=0, \quad$ where $\delta_{m}=\max _{1 \leq i \leq m}\left\{t_{i}-t_{i-1}: \quad i=1,2, \ldots, m\right\}$.

The distribution of the knots $\{t\}_{i=0}^{m}$ is called to be more-or-less uniform if (see e.g. [9]):

Definition 1.2 The sampling $\mathcal{T}_{m o l}$ is more-or-less uniform if for some constants $0<K_{l} \leq K_{u}$ and sufficiently large $m$ the inequalities:

$$
\frac{K_{l}}{m} \leq t_{i}-t_{i-1} \leq \frac{K_{u}}{m}
$$

hold for all $i=1,2, \ldots, m$. Equivalently, more-or-less uniformity stipulates the existence of some constant $0<\beta \leq 1$ satisfying $\beta \delta_{m} \leq t_{i}-t_{i-1} \leq \delta_{m}$, for all $i=1,2, \ldots, m$.

In this work, the missing knots $\left\{t_{i}\right\}_{i=0}^{m}$ are estimated by $\left\{\hat{t}_{i}^{\lambda}\right\}_{i=0}^{m}$ according to the exponential parameterization (see e.g. [18]):

$$
\hat{t}_{0}^{\lambda}=0 \text { and } \hat{t}_{i}^{\lambda}=\hat{t}_{i-1}^{\lambda}+\left\|q_{i}-q_{i-1}\right\|^{\lambda}
$$

for $i=1,2, \ldots, m$, with superscript $\lambda \in[0,1]$ representing a free parameter. ${ }^{1}$ Visibly the condition $q_{i} \neq q_{i+1}$ enforces $\hat{t}_{i}^{\lambda}<\hat{t}_{i+1}^{\lambda}$. The case $\lambda=0$ yields uniform knots $\hat{t}_{i}^{0}=i$, whereas $\lambda=1$ results in cumulative chord parameterization (see e.g. $[18,19])$ for which we have:

$\hat{t}_{i}^{1}=\hat{t}_{i-1}^{1}+\left\|q_{i}-q_{i-1}\right\|$.

Definition 1.3 Consider a family $\left\{f_{\delta_{m}}, \delta_{m}>0\right\}$ of functions $f_{\delta_{m}}:[0, T] \rightarrow \mathbb{E}$. We say that $f_{\delta_{m}}$ is of order $O\left(\delta_{m}^{\alpha}\right)$ (denoted as $f_{\delta_{m}}=O\left(\delta_{m}^{\alpha}\right)$ ), if there is a constant $K>0$ such that, for some $\bar{\delta}>0$ the inequality $\left|f_{\delta_{m}}(t)\right| \leq K \delta_{m}^{\alpha}$ holds for all $\delta_{m} \in(0, \bar{\delta})$, uniformly over $[0, T]$. In case of vector-valued functions $F_{\delta_{m}}:[0, T] \rightarrow \mathbb{E}^{n}$ by $F_{\delta_{m}}=O\left(\delta_{m}^{\alpha}\right)$ it is meant that $\left\|F_{\delta_{m}}\right\|=O\left(\delta_{m}^{\alpha}\right)$.

Definition 1.4 Let $f_{i}:\left[a_{i}, b_{i}\right] \rightarrow \mathbb{E}^{n}$ with $0 \leq a_{i} \leq b_{i}$, be given for $0 \leq i \leq k-1$. By the track-sum of the family of functions $\left\{f_{i}\right\}_{i=0}^{k-1}$ we understand the function $f:[0, \hat{T}] \rightarrow \mathbb{E}^{n}$, where $\hat{T}=\sum_{i=0}^{k-1}\left(b_{i}-a_{i}\right)$ satisfying (for $0 \leq k \leq k_{0}-2$ ): $f(t)=f_{0}\left(t+a_{0}\right)$ for $t \in\left[0, \hat{T}_{0}\right]$ (with $\left.\hat{T}_{0}=b_{0}-a_{0}\right)$ and $f(t)=f_{k+1}\left(t+a_{k+1}-T_{k}\right.$ ), for $t \in\left[\hat{T}_{k}, \hat{T}_{k+1}\right]$ with $\hat{T}_{k+1}=\hat{T}_{k}+b_{k+1}-a_{k+1}$.

Recall also the following notion (see [1]):

\footnotetext{
${ }^{1}$ From now on we omit $\lambda$ in our notation (unless needed otherwise).
} 
Definition 1.5 Let $\tau=\left\{\tau_{i}\right\}_{i=1}^{k}$ be a sequence of sites not necessarily distinct. We say that the function p agrees with the function $g$ at $\tau$ provided that, for every site $\xi$ that occurs $l$ times in the sequence $\tau_{1}, \ldots, \tau_{k}, p$ and $g$ agree $l$-fold at $\xi$, that is:

$p^{(i-1)}(\xi)=g^{(i-1)}(\xi)$,

for $i=1, \ldots, l$.

Newton osculatory interpolation theorem [1] for possibly repeated ordered sites $\tau_{1} \leq \tau_{2} \leq \ldots \leq \tau_{k}$ reads:

Theorem 1.6 If $g \in C^{n}$ class and $\tau=\left\{\tau_{i}\right\}_{i=1}^{k}$ is a sequence of $k$ arbitrary sites (not necessarily distinct), then there exists exactly one polynomial $p_{n}$ of order $n$ that agrees with $g$ at $\tau$ equal to:

$p_{n}(t)=\sum_{i=1}^{n}\left(t-\tau_{1}\right) \cdots\left(t-\tau_{i-1}\right) g\left[\tau_{1}, \ldots, \tau_{i}\right]$,

where $g\left[\tau_{1}\right]=g\left(\tau_{1}\right)$ and if $\tau_{i}=\ldots=\tau_{i+j}$ and $g \in C^{j}$ then

$g\left[\tau_{i}, \ldots, \tau_{i+j}\right]=\frac{g^{(j)}\left(\tau_{i}\right)}{j !}$,

or otherwise if $\tau_{i}<\tau_{i+j}$ then

$g\left[\tau_{i}, \ldots, \tau_{i+j}\right]=\frac{g\left[\tau_{i+1}, \ldots, \tau_{i+j}\right]-g\left[\tau_{i}, \ldots, \tau_{i+j-1}\right]}{\tau_{i+j}-\tau_{i}}$.

In addition, for all $t$ we have:

$g(t)=p_{n}(t)+\left(t-\tau_{1}\right) \cdots\left(t-\tau_{n}\right) g\left[\tau_{1}, \ldots, \tau_{n}, t\right]$.

Section 2 recalls the construction of the modified Hermite interpolant $\hat{\gamma}^{H}:[0, \hat{T}] \rightarrow \mathbb{E}^{n}$. The respective asymptotics to approximate $\gamma \in C^{4}$ (sampled admissibly—see Definition 1.1) by $\hat{\gamma}^{H}$ is so-far addressed only for $\lambda=1$ appearing in (1.2)—see [7,8]. More specifically, a fast sharp quartic convergence order $\alpha(1)=4$ (see Definition 1.3) in estimating $\gamma$ by $\hat{\gamma}^{H} \circ \phi^{H}$ is proved. Note that both $\hat{\gamma}^{H}$ and $\phi^{H}$ are precisely determined in Sect. 2 below.

Remark 1 For a given interpolation scheme $\hat{\gamma}$ based on reduced data $Q_{m}$ and fixed estimates $\hat{\mathcal{T}}$ of the unknown knots $\mathcal{T}$ (and subject to some selected mapping $\phi:[0, T] \rightarrow[0, \hat{T}]$ ) we say that asymptotics $\gamma-\hat{\gamma} \circ \phi=O\left(\delta_{m}^{\alpha}\right)$ over $[0, T]$ is sharp within the prescribed family of curves $\gamma \in \mathcal{J}$ and family of samplings $\mathcal{T} \in \mathcal{K}$, if for some $\gamma \in \mathcal{J}$ and some some sampling from $\mathcal{K}$, there exists $t^{*} \in[0, T]$ and some positive constant $K$ such that $\left\|\gamma\left(t^{*}\right)-(\hat{\gamma} \circ \phi)\left(t^{*}\right)\right\|=K \delta_{m}^{\alpha}+O\left(\delta_{m}^{\beta}\right)$, where $\beta>\alpha$. For example, for sharp quartic order from (3.1): $\mathcal{J}=C^{4}$, the admissible samplings (1.1) forms $\mathcal{K}, \hat{\gamma}=\hat{\gamma}^{H}$ with $\phi=\phi^{H}$ and $\hat{\mathcal{T}}$ is defined according to (1.2), where $\lambda=1$.

This paper's main contribution (see Sect. 3) extends the above result to the remaining cases of $\lambda \in[0,1$ ) controlling (1.2) with $\mathcal{K}$ representing more-or-less uniform samplings—see Definition 1.2.

In Sect. 4 an abrupt deceleration effect in asymptotic orders dropping from $\alpha(1)=4$ to $\alpha(\lambda)=1$, for all $\lambda \in[0,1)$, is analytically proved.

Section 5 supplements the main theoretical contribution of this work established in Theorem 3.2. The numerical tests (conducted in Mathematica) verify in affirmative the sharpness of the asymptotics from (3.2) - see also Remark 1. In addition, more-or-less uniformity of $\mathcal{T}$ assumed in Theorem 3.2 is also justified numerically as indispensable.

The last Sect. 6 concludes this paper and reiterates its main gist. The alternative spline schemes $\hat{\gamma}$ to approximate $\gamma$ and based on $Q_{m}$ and (1.2) are briefly recalled in the context of underlying asymptotics. Some applications and open problems are also outlined. 


\section{Modified Hermite Interpolation}

Interpolating $Q_{m}$ by a piecewise Lagrange cubic $\hat{\gamma}$ (see e.g. [1]) defined as a track-sum of cubics $\hat{\gamma}_{i}:\left[\hat{t}_{i}, \hat{t}_{i+3}\right] \rightarrow \mathbb{E}^{n}$ (where $\gamma_{i}\left(t_{i+k}\right)=q_{i+k}$ for $k=0,1,2,3$ ) yields generically non-smooth curve $\hat{\gamma}$ at all junction points $q_{3}, q_{6}, \ldots, q_{m-3}$. In order to rectify this deficiency, the data points $Q_{m}$ can be first supplemented with some good estimates of the unsupplied exact velocities i.e. with some $v\left(q_{i}\right) \approx \dot{\gamma}\left(t_{i}\right)$ (each pair in $\mathbb{R}^{n}$ ). In sequel, a $C^{1}$ Hermite interpolation (see e.g. [1]) can now be applied to each pair of points $\left(q_{i}, q_{i+1}\right)$ and the respective velocities $\left(v\left(q_{i}\right), v\left(q_{i+1}\right)\right)$. The precise definition of $\hat{\gamma}^{H}$ goes along the following pattern (see [7,8]):

- Construct Lagrange cubics $\hat{\gamma}_{i}:\left[\hat{t}_{i}, \hat{t}_{i+3}\right] \rightarrow \mathbb{E}^{n}$ satisfying $\hat{\gamma}\left(\hat{t}_{i+j}\right)=q_{i+j}$ with $j=0,1,2,3$ and $i=$ $0,1, \ldots, m-3$.

- Assign the velocities $\left\{v\left(q_{i}\right)=\hat{\gamma}_{i}^{\prime}\left(\hat{t}_{i}\right)\right\}_{i=0}^{m-3}$ as the respective approximations of $\left\{\dot{\gamma}\left(t_{i}\right)\right\}_{i=0}^{m-3}$.

- Note that for the last three points $\left(q_{m-2}, q_{m-1}, q_{m}\right)$ the respective velocities $\left\{v\left(q_{j}\right)\right\}_{j=m-3}^{m}$ are analogously computed with the aid of reversing the last $\left\{\hat{t}_{i}\right\}_{i=0}^{m-5}$ —for more details see [8].

- Define Hermite cubics $\hat{\gamma}_{i}^{H}:\left[\hat{t}_{i}, \hat{t}_{i+1}\right] \rightarrow \mathbb{E}^{n}$ satisfying (for $i=0,1, \ldots, m-1$ and $k=0,1$ ):

$\hat{\gamma}_{i}^{H}\left(\hat{t}_{i+k}\right)=q_{i+k}$ and $\hat{\gamma}_{i}^{H \prime}\left(\hat{t}_{i+k}\right)=\hat{\gamma}_{i+k}^{\prime}\left(\hat{t}_{i+k}\right)$.

The Newton interpolation formula (1.3), over each sub-interval $\hat{I}_{i}=\left[\hat{t}_{i}, \hat{t}_{i+1}\right]$, yields:

$$
\begin{aligned}
\hat{\gamma}_{i}^{H}(\hat{t})= & \hat{\gamma}_{i}^{H}\left[\hat{t}_{i}\right]+\hat{\gamma}_{i}^{H}\left[\hat{t}_{i}, \hat{t}_{i}\right]\left(\hat{t}-\hat{t}_{i}\right)+\hat{\gamma}_{i}^{H}\left[\hat{t}_{i}, \hat{t}_{i}, \hat{t}_{i+1}\right]\left(\hat{t}-\hat{t}_{i}\right)^{2} \\
& +\hat{\gamma}_{i}^{H}\left[\hat{t}_{i}, \hat{t}_{i}, \hat{t}_{i+1}, \hat{t}_{i+1}\right]\left(\hat{t}-\hat{t}_{i}\right)^{2}\left(\hat{t}-\hat{t}_{i+1}\right) .
\end{aligned}
$$

- The curve $\hat{\gamma}^{H}$ (coined as modified Hermite cubic — see [7]) represents a track-sum of $\left\{\hat{\gamma}_{i}^{H}\right\}_{i=0}^{m-1}$.

To estimate the difference between $\gamma$ and $\hat{\gamma}^{H}$ with the aid of the infinity norm (see Definition 1.3) the respective domains of both curves i.e. $[0, T]$ and $[0, \hat{T}]$ should coincide so that interpolation conditions $\hat{\gamma}\left(\hat{t}_{i}\right)=\gamma\left(t_{i}\right)=q_{i}$ are preserved. In doing so, some mapping $\phi_{i}:\left[t_{i}, t_{i+1}\right] \rightarrow\left[\hat{t}_{i}, \hat{t}_{i+1}\right]$ is needed with $\phi$ defined as as track-sum of $\left\{\phi_{i}\right\}_{i=0}^{m-1}$. At best it should constitute a genuine reparameterization of $I_{i}=\left[t_{i}, t_{i+1}\right]$ into $\hat{I}_{i}$, e.g. if length $d(\gamma)$ of $\gamma$ is also to be estimated by $d(\hat{\gamma})$ (see e.g. $[5,16]$ ). In contrast with the latter, some other applications (e.g. path modelling and planning in robotics or drone navigation) may require multiple or single loops (see e.g. [11]).

A similar approach as used above can be adopted to generate $\phi_{i}=\phi_{i}^{H}: I_{i} \rightarrow \hat{I}_{i}$. Indeed, the following procedure for the construction of $\phi^{H}$ is proposed in [7]:

- Define first family of Lagrange cubics $\psi_{i}:\left[t_{i}, t_{i+3}\right] \rightarrow\left[\hat{t}_{i}, \hat{t}_{i+3}\right]$ for $i=0,1, \ldots, m-3$ satisfying the interpolation conditions (with $j=0,1,2,3$ ):

$\psi_{i}\left(t_{i+j}\right)=\hat{t}_{i+j}$

- Construct next Hermite cubics $\phi_{i}^{H}:\left[t_{i}, t_{i+1}\right] \rightarrow\left[\hat{t}_{i}, \hat{t}_{i+1}\right]$ satisfying (for $\left.k=0,1\right)$ :

$\phi_{i}^{H}\left(t_{i+k}\right)=\hat{t}_{i+k} \quad$ and $\quad \dot{\phi}_{i}^{H}\left(t_{i+k}\right)=\dot{\psi}_{i+k}\left(t_{i+k}\right)$.

For the last three knots the respective derivatives can be found as for $\hat{\gamma}^{H}$.

- Again here $\phi=\phi^{H}$ represents a track-sum of $\left\{\phi_{i}^{H}\right\}_{i=0}^{m-1}$.

\section{The Main Result}

The following sharp result holding for $\lambda=1$ (see Remark 1) is established in [7,8]:

Theorem 3.1 Let $\gamma$ be a regular $C^{4}([0, T])$ curve in $\mathbb{E}^{n}$ sampled admissibly (see $\left.(1.1)\right)$. Given reduced data $Q_{m}$ with $\left\{\hat{t}_{i}^{\lambda=1}\right\}_{i=0}^{m}$ determined by $\lambda=1$ in (1.2), there exists a piecewise-cubic $C^{1}$ reparameterization $\phi^{H}:[0, T] \rightarrow[0, \hat{T}]$ (a track-sum of $\left\{\phi_{i}^{H}\right\}_{i=0}^{m-1}$ ) such that (uniformly over $I_{i}$ ):

$\gamma-\hat{\gamma}^{H} \circ \phi^{H}=O\left(\delta_{m}^{4}\right)$. 
This paper complements (3.1) to the remaining $\lambda \in[0,1)$ and $\mathcal{T}$ more-or-less uniform. Contrasting Theorem 3.1, some choices of $\lambda \in[0,1)$, reduced data $Q_{m}$ and $\gamma$ sampled more-or-less uniformly may not yield $\phi_{i}^{H}$ and $\psi_{i}$ as genuine reparameterizations (see also [9-11]). In particular, to cover the cases of $\left[\hat{t}_{i}, \hat{t}_{i+3}\right] \subset \psi_{i}\left(\left[t_{i}, t_{i+3}\right]\right)$ and of $\hat{I}_{i} \subset \phi_{i}^{H}\left(I_{i}\right)$ the domains of cubics $\hat{\gamma}_{i}$ and $\hat{\gamma}_{i}^{H}$ are naturally extended to $\mathbb{R}$. Such cubics are denoted here by $\check{\gamma}_{i}$ and $\check{\gamma}_{i}^{H}$ and satisfy $\left.\check{\gamma}_{i}\right|_{\left[t_{i}, t_{i+3}\right]}=\hat{\gamma}_{i}$ and $\left.\check{\gamma}_{i}^{H}\right|_{\left[t_{i}, t_{i+1}\right]}=\hat{\gamma}_{i}^{H}$, respectively. The main result of this paper reads as:

Theorem 3.2 Let $\gamma$ be a regular $C^{4}([0, T])$ curve in $\mathbb{E}^{n}$ sampled more-or-less uniformly (1.2). For reduced data $Q_{m}$ and $\left\{\hat{t}_{i}^{\lambda}\right\}_{i=0}^{m}$ defined by $(1.2)$ (with $\left.\lambda \in[0,1)\right)$ there exists a piecewise-cubic $C^{1}$ mapping $\phi^{H}:[0, T] \rightarrow[0, \hat{T}]$ such that (uniformly over $[0, T]$ ), for $i=0,1, \ldots, m-1$ we have:

$\gamma-\check{\gamma}_{i}^{H} \circ \phi_{i}^{H}=O\left(\delta_{m}\right)$.

We remark here that if $\phi_{i}^{H}\left(I_{i}\right) \subset \hat{I}_{i}$ (which occurs e.g. if $\phi_{i}$ forms a reparameterization) the interpolant $\check{\gamma}_{i}^{H}$ can be replaced with $\hat{\gamma}_{i}^{H}$ in (3.2). However, even if $\phi_{i}^{H}: I_{i} \rightarrow \hat{I}_{i}$ it may still not form a reparameterization and the trajectory of $\hat{\gamma}_{i}^{H} \circ \phi_{i}^{H}$ gets extra loops (which also occurs if $\hat{I}_{i} \subset \phi_{i}^{H}\left(I_{i}\right)$ and $\hat{I}_{i} \neq \phi_{i}^{H}\left(I_{i}\right)$ ). The curve $\left.\check{\gamma}_{i}\right|_{\left[t_{i}, t_{i+3}\right]}=\hat{\gamma}_{i}$ is used below in proving Theorem 3.2.

The next section proves Theorem 3.2. The sharpness of (3.2) is numerically verified in Sect. 5.

\section{The Proof of Theorem 3.2}

The proof of Theorem 3.2 is given for an arbitrary $\lambda \in[0,1)$. The case of $\lambda=1$ in (1.2) addressed in Theorem 3.1 is already justified in $[7,8]$.

Proof In order to extract the underlying asymptotics of $f=\gamma-\check{\gamma}^{H} \circ \phi^{H}$ (uniformly over [0,T]) it suffices to examine the latter for any $f_{i}=\left.f\right|_{I_{i}}=\gamma-\check{\gamma}_{i}^{H} \circ \phi_{i}^{H}$ over the respective sub-interval $I_{i}$ (here $i=1,2, \ldots, m-1$ ). The first step splits the asymptotics of each $f_{i}$ into separate components.

\subsection{Step 1: Decomposition of the Asymptotics for $f_{i}$}

Observe that each function $f_{i}$ (over the respective $I_{i}$ ) satisfies:

$$
f_{i}(t)=\gamma(t)-\left(\check{\gamma}_{i} \circ \psi_{i}\right)(t)+\left(\check{\gamma}_{i} \circ \psi_{i}\right)(t)-\left(\check{\gamma}_{i}^{H} \circ \phi_{i}^{H}\right)(t),
$$

where $\psi_{i}$ is the Lagrange cubic (see Sect. 2). As $\gamma \in C^{4}$ and $\lambda \in[0,1)$ by [12] (or [9]) we have:

$\gamma-\check{\gamma}_{i} \circ \psi_{i}=O\left(\delta_{m}\right)$

and thus to justify $f_{i}=O\left(\delta_{m}\right)$ it suffices to show that the second component in (4.1) is also of order $O\left(\delta_{m}\right)$ (uniformly over $I_{i}$ ). Newton interpolation formula (1.5) (with the remainder) applied to the up to the 9-th order polynomial $\rho_{i}=\check{\gamma}_{i} \circ \psi_{i}-\check{\gamma}_{i}^{H} \circ \phi_{i}^{H}$ (over each $I_{i}$ ) yields:

$$
\begin{gathered}
\rho_{i}(t)=\rho_{i}\left[t_{i}\right]+\rho_{i}\left[t_{i}, t_{i}\right]\left(t-t_{i}\right)+\rho_{i}\left[t_{i}, t_{i}, t_{i+1}\right]\left(t-t_{i}\right)^{2}+\rho_{i}\left[t_{i}, t_{i}, t_{i+1}, t_{i+1}\right]\left(t-t_{i}\right)^{2}\left(t-t_{i+1}\right) \\
+\left(t-t_{i}\right)^{2}\left(t-t_{i+1}\right)^{2} \rho_{i}\left[t, t_{i}, t_{i}, t_{i+1}, t_{i+1}\right] .
\end{gathered}
$$

Coupling (2.1) and (2.3) together with (2.2) and (2.4) renders $\rho_{i}\left[t_{i}\right]=\overrightarrow{0}$ (and by Theorem 1.6) yields $\rho_{i}\left[t_{i}, t_{i}\right]=$ $\rho_{i}^{\prime}\left(t_{i}\right)=\overrightarrow{0}$. The latter together with Theorem 1.6 leads to $\rho_{i}\left[t_{i}, t_{i+1}\right]=\overrightarrow{0}$. Hence, again by Theorem 1.6 we obtain:

$\rho_{i}\left[t_{i}, t_{i}, t_{i+1}\right]=\frac{\rho_{i}\left[t_{i}, t_{i+1}\right]-\rho_{i}\left[t_{i}, t_{i}\right]}{t_{i+1}-t_{i}}=\overrightarrow{0}$. 
Thus (4.3) reduces into:

$$
\rho_{i}(t)=\rho_{i}\left[t_{i}, t_{i}, t_{i+1}, t_{i+1}\right]\left(t-t_{i}\right)^{2}\left(t-t_{i+1}\right)+\left(t-t_{i}\right)^{2}\left(t-t_{i+1}\right)^{2} \rho_{i}\left[t_{i}, t_{i}, t_{i+1}, t_{i+1}, t\right] .
$$

Hence as $\rho_{i}\left[t_{i}, t_{i+1}\right]=\rho_{i}\left[t_{i}, t_{i}, t_{i+1}\right]=\overrightarrow{0}$ and $\dot{\rho}=\check{\gamma}_{i}^{\prime} \dot{\psi}_{i}-\check{\gamma}_{i}^{H \prime} \dot{\phi}_{i}^{H}$ by Theorem 1.6 we obtain:

$$
\begin{aligned}
\rho_{i}\left[t_{i}, t_{i}, t_{i+1}, t_{i+1}\right] & =\frac{\rho_{i}\left[t_{i}, t_{i+1}, t_{i+1}\right]}{t_{i+1}-t_{i}}=\frac{\dot{\rho}\left(t_{i+1}\right)}{\left(t_{i+1}-t_{i}\right)^{2}} \\
& =\frac{\check{\gamma}_{i}^{\prime}\left(\hat{t}_{i+1}\right) \dot{\psi}_{i}\left(t_{i+1}\right)-\dot{\gamma}\left(t_{i+1}\right)+\dot{\gamma}\left(t_{i+1}\right)-\check{\gamma}_{i}^{H \prime}\left(\hat{t}_{i+1}\right) \dot{\phi}_{i}^{H}\left(t_{i+1}\right)}{\left(t_{i+1}-t_{i}\right)^{2}} .
\end{aligned}
$$

Before proceeding further recall that for $k=0,1$ by [12] we have (see also [9]):

$\dot{\psi}_{i+k}=O\left(\delta_{m}^{\lambda-1}\right), \quad \ddot{\psi}_{i+k}=O\left(\delta_{m}^{\lambda-2}\right), \quad$ and $\quad \dddot{\psi}_{i+k}=O\left(\delta_{m}^{\lambda-3}\right)$,

and

$$
\check{\gamma}_{i+k}^{\prime}=O\left(\delta_{m}^{1-\lambda}\right), \quad \check{\gamma}_{i+k}^{\prime \prime}=O\left(\delta_{m}^{1-2 \lambda}\right), \quad \text { and } \quad \check{\gamma}_{i+k}^{\prime \prime \prime}=O\left(\delta_{m}^{1-3 \lambda}\right),
$$

both holding over $I_{i+k}$ and over $\psi_{i+k}\left(I_{i+k}\right)$, respectively. Hence by (4.7) and (4.8) we obtain:

$\dot{\gamma}\left(t_{i+1}\right)-\check{\gamma}_{i}^{\prime}\left(\hat{t}_{i+1}\right) \dot{\psi}_{i}\left(t_{i+1}\right)=O(1)+O\left(\delta_{m}^{1-\lambda}\right) \cdot O\left(\delta_{m}^{\lambda-1}\right)=O(1)$.

Similarly, coupling together (2.1) and (2.4) with (4.7) and (4.8) leads to:

$\dot{\gamma}\left(t_{i+1}\right)-\check{\gamma}_{i}^{H \prime}\left(\hat{t}_{i+1}\right) \dot{\phi}_{i}^{H}\left(t_{i+1}\right)=\dot{\gamma}\left(t_{i+1}\right)-\check{\gamma}_{i+1}^{\prime}\left(\hat{t}_{i+1}\right) \dot{\psi}_{i+1}\left(t_{i+1}\right)=O(1)$.

Thus, combining (4.9) with (4.10) reduces (4.6) into: $\rho_{i}\left[t_{i}, t_{i}, t_{i+1}, t_{i+1}\right]=\left(t_{i+1}-t_{i}\right)^{-2} O(1)$. Hence as $t \in I_{i}$, the latter simplifies (4.5) into:

$\rho_{i}(t)=O\left(\delta_{m}\right)+\left(t-t_{i}\right)^{2}\left(t-t_{i+1}\right)^{2} \rho_{i}\left[t_{i}, t_{i}, t_{i+1}, t_{i+1}, t\right]$.

We examine now the asymptotics of the second term $\rho_{i}\left[t_{i}, t_{i}, t_{i+1}, t_{i+1}, t\right]$ appearing in (4.11). By standard property of the divided differences (see e.g. [1]) each component of $\rho_{i}$ (with $1 \leq j \leq n$ ) reads as:

$\rho_{i}\left[t_{i}, t_{i}, t_{i+1}, t_{i+1}, t\right]_{j}=\frac{\rho_{i}^{(4)}\left(\tilde{t}_{j}^{i}\right)}{4 !}$,

for some $\tilde{t}_{j}^{i} \in\left[t_{i}, t_{i+1}\right]$ Hence, by the chain rule the expression $4 ! \rho_{i}\left[t, t_{i}, t_{i}, t_{i+1}, t_{i+1}\right]_{j}$ (here the index $j$ in $\rho_{i}$ and in (4.12) stands for the $j$-th component, with $1 \leq j \leq n$ ) at $\tilde{t}_{j}^{i}$ is equal to:

$$
6\left(\dot{\psi}_{i}\right)^{2} \ddot{\psi}_{i} \check{\gamma}_{i, j}^{\prime \prime \prime}+3\left(\ddot{\psi}_{i}\right)^{2} \check{\gamma}_{i, j}^{\prime \prime}+4 \dot{\psi}_{i} \dddot{\psi}_{i} \check{\gamma}_{i, j}^{\prime \prime}-6\left(\dot{\phi}_{i}^{H}\right)^{2} \ddot{\phi}_{i}^{H} \check{\gamma}_{i, j}^{H \prime \prime \prime}-3\left(\ddot{\phi}_{i}^{H}\right)^{2} \check{\gamma}_{i, j}^{H \prime \prime}-4 \dot{\phi}_{i}^{H} \ddot{\phi}_{i}^{H} \check{\gamma}_{i, j}^{H \prime \prime},
$$

with all derivatives of $\psi_{i}$ and $\phi_{i}^{H}$ evaluated at $\tilde{t}_{j}^{i}$ and all derivatives of $\check{\gamma}_{i, j}$ (or $\check{\gamma}_{i, j}^{H}$ ) evaluated at $\psi_{i}\left(\tilde{t}_{j}^{i}\right.$ ) (or at $\left.\phi_{i}^{H}\left(\tilde{t}_{j}^{i}\right)\right)$. To determine the asymptotics governing (4.12), given (4.7) and (4.8), it suffices to find the asymptotics for the derivatives of $\check{\gamma}_{i}^{H}$ (over $\phi_{i}^{H}\left(I_{i}\right)$ ) and of $\phi_{i}^{H}$ (over $I_{i}$ ), respectively.

\subsection{Step 2: Asymptotics for Derivatives of the Mapping $\phi_{i}^{H}$}

Coupling together Theorem 1.6, (2.3), (2.4) and (4.7) leads to:

$$
\begin{aligned}
\phi_{i}^{H}\left[t_{i}, t_{i}\right] & =\dot{\psi}_{i}\left(t_{i}\right)=O\left(\delta_{m}^{\lambda-1}\right), \\
\phi_{i}^{H}\left[t_{i}, t_{i}, t_{i+1}\right] & =\frac{\psi_{i}\left(t_{i+1}\right)-\psi_{i}\left(t_{i}\right)}{\left(t_{i+1}-t_{i}\right)^{2}}-\frac{\dot{\psi}_{i}\left(t_{i}\right)}{t_{i+1}-t_{i}}=\psi_{i}\left[t_{i}, t_{i}, t_{i+1}\right]=O\left(\ddot{\psi}_{i}\right) \\
& =O\left(\delta_{m}^{\lambda-2}\right) .
\end{aligned}
$$


Furthermore, by (4.7) as $\dot{\psi}_{i}\left(t_{i+1}\right)=O\left(\delta_{m}^{\lambda-1}\right)$ and $\dot{\psi}_{i+1}\left(t_{i+1}\right)=O\left(\delta_{m}^{\lambda-1}\right)$ we have $\dot{\psi}_{i+1}\left(t_{i+1}\right)=\dot{\psi}_{i}\left(t_{i}\right)+O\left(\delta_{m}^{\lambda-1}\right)$. The latter combined with more-or-less uniformity, (2.3), (2.4) and (4.7) results into (and also by Theorem 1.6):

$$
\begin{aligned}
\phi_{i}^{H}\left[t_{i}, t_{i}, t_{i+1}, t_{i+1}\right] & =\frac{\dot{\phi}_{i}^{H}\left(t_{i+1}\right)-\phi_{i}^{H}\left[t_{i}, t_{i+1}\right]}{\left(t_{i+1}-t_{i}\right)^{2}}-\frac{\phi_{i}^{H}\left[t_{i}, t_{i}, t_{i+1}\right]}{t_{i+1}-t_{i}}=\frac{\dot{\psi}_{i+1}\left(t_{i+1}\right)-\psi_{i}\left[t_{i}, t_{i+1}\right]}{\left(t_{i+1}-t_{i}\right)^{2}}-\frac{\psi_{i}\left[t_{i}, t_{i}, t_{i+1}\right]}{t_{i+1}-t_{i}} \\
& =\frac{\dot{\psi}_{i}\left(t_{i+1}\right)-\psi_{i}\left[t_{i}, t_{i+1}\right]}{\left(t_{i+1}-t_{i}\right)^{2}}-\frac{\psi_{i}\left[t_{i}, t_{i}, t_{i+1}\right]}{t_{i+1}-t_{i}}+\frac{O\left(\delta_{m}^{\lambda-1}\right)}{\left(t_{i+1}-t_{i}\right)^{2}} \\
& =\psi_{i}\left[t_{i}, t_{i}, t_{i+1}, t_{i+1}\right]+O\left(\delta_{m}^{\lambda-3}\right)=O\left(\ddot{\psi}_{i}\right)+O\left(\delta_{m}^{\lambda-3}\right) \\
& =O\left(\delta_{m}^{\lambda-3}\right) .
\end{aligned}
$$

Newton interpolation formula (1.3) (applied over each $I_{i}$ ) yields:

$\phi_{i}^{H}(t)=\phi_{i}^{H}\left[t_{i}\right]+\phi_{i}^{H}\left[t_{i}, t_{i}\right]\left(t-t_{i}\right)+\phi_{i}^{H}\left[t_{i}, t_{i}, t_{i+1}\right]\left(t-t_{i}\right)^{2}+\phi_{i}^{H}\left[t_{i}, t_{i}, t_{i+1}, t_{i+1}\right]\left(t-t_{i}\right)^{2}\left(t-t_{i+1}\right)$,

which in turn combined with (4.13) and (4.15) leads to:

$\dot{\phi}_{i}^{H}=O\left(\delta_{m}^{\lambda-1}\right), \quad \ddot{\phi}_{i}^{H}=O\left(\delta_{m}^{\lambda-2}\right)$ and $\dddot{\phi}_{i}^{H}=O\left(\delta_{m}^{\lambda-3}\right)$.

\subsection{Step 3: Asymptotics for Derivatives of the Interpolant $\check{\gamma}_{i}^{H}$}

We examine now the asymptotics of the respective divided differences for $\check{\gamma}^{H}$. In doing so, note that by (2.1) and (4.8) the following holds (upon using again Theorem 1.6):

$$
\begin{aligned}
\check{\gamma}_{i}^{H}\left[\hat{t}_{i}, \hat{t}_{i}\right] & =\check{\gamma}_{i}^{\prime}\left(\hat{t}_{i}\right)=O\left(\delta_{m}^{1-\lambda}\right) . \\
\check{\gamma}_{i}^{H}\left[\hat{t}_{i}, \hat{t}_{i}, \hat{t}_{i+1}\right] & =\frac{\check{\gamma}_{i}^{H}\left(\hat{t}_{i+1}\right)-\check{\gamma}_{i}^{H}\left(\hat{t}_{i}\right)}{\left(\hat{t}_{i+1}-\hat{t}_{i}\right)^{2}}-\frac{\check{\gamma}_{i}^{H \prime}\left(\hat{t}_{i}\right)}{\hat{t}_{i+1}-\hat{t}_{i}}=\frac{\check{\gamma}_{i}\left(\hat{t}_{i+1}\right)-\check{\gamma}_{i}\left(\hat{t}_{i}\right)}{\left(\hat{t}_{i+1}-\hat{t}_{i}\right)^{2}}-\frac{\check{\gamma}_{i}^{\prime}\left(\hat{t}_{i}\right)}{\hat{t}_{i+1}-\hat{t}_{i}} \\
& =\check{\gamma}_{i}\left[\hat{t}_{i}, \hat{t}_{i}, \hat{t}_{i+1}\right]=O\left(\check{\gamma}_{i}^{\prime \prime}\right) \\
& =O\left(\delta_{m}^{1-2 \lambda}\right) .
\end{aligned}
$$

Coupling (2.1) and (4.8) again with Theorem 1.6 renders:

$$
\begin{aligned}
\check{\gamma}_{i}^{H}\left[\hat{t}_{i}, \hat{t}_{i}, \hat{t}_{i+1}, \hat{t}_{i+1}\right] & =\frac{\check{\gamma}_{i}^{H}\left[\hat{t}_{i}, \hat{t}_{i+1}, \hat{t}_{i+1}\right]-\check{\gamma}_{i}^{H}\left[\hat{t}_{i}, \hat{t}_{i}, \hat{t}_{i+1}\right]}{\hat{t}_{i+1}-\hat{t}_{i}}=\frac{\check{\gamma}_{i}^{H}\left[\hat{t}_{i}, \hat{t}_{i+1}, \hat{t}_{i+1}\right]-\check{\gamma}_{i}\left[\hat{t}_{i}, \hat{t}_{i}, \hat{t}_{i+1}\right]}{\hat{t}_{i+1}-\hat{t}_{i}} \\
& =\frac{\frac{\check{\gamma}_{i+1}^{\prime}\left(\hat{t}_{i+1}\right)-\check{\gamma}_{i}\left[\hat{t}_{i}, \hat{t}_{i+1}\right]}{\hat{t}_{i+1}-\hat{t}_{i}}-\check{\gamma}_{i}\left[\hat{t}_{i}, \hat{t}_{i}, \hat{t}_{i+1}\right]}{\hat{t}_{i+1}-\hat{t}_{i}} .
\end{aligned}
$$

Since $\check{\gamma}_{i+1}\left(\hat{t}_{i+1}\right)=O\left(\delta_{m}^{1-\lambda}\right)$ and $\check{\gamma}_{i}\left(\hat{t}_{i+1}\right)=O\left(\delta_{m}^{1-\lambda}\right)$ (see (4.8)) the following holds $\check{\gamma}_{i+1}\left(\hat{t}_{i+1}\right)=\check{\gamma}_{i}\left(\hat{t}_{i+1}\right)+$ $O\left(\delta_{m}^{1-\lambda}\right)$ which by Theorem 1.6 ultimately expands (4.18) into

$$
\begin{aligned}
\check{\gamma}_{i}^{H}\left[\hat{t}_{i}, \hat{t}_{i}, \hat{t}_{i+1}, \hat{t}_{i+1}\right] & =\frac{\frac{\check{\gamma}_{i}^{\prime}\left(\hat{t}_{i+1}\right)-\check{\gamma}_{i}\left[\hat{t}_{i}, \hat{t}_{i+1}\right]}{\hat{t}_{i+1}-\hat{t}_{i}}-\check{\gamma}_{i}\left[\hat{t}_{i}, \hat{t}_{i}, \hat{t}_{i+1}\right]}{\hat{t}_{i+1}-\hat{t}_{i}}+\frac{O\left(\delta_{m}^{1-\lambda}\right)}{\left(\hat{t}_{i+1}-\hat{t}_{i}\right)^{2}} \\
& =\check{\gamma}_{i}\left[\hat{t}_{i}, \hat{t}_{i}, \hat{t}_{i+1}, \hat{t}_{i+1}\right]+\frac{O\left(\delta_{m}^{1-\lambda}\right)}{\left(\hat{t}_{i+1}-\hat{t}_{i}\right)^{2}}=O\left(\check{\gamma}_{i}^{\prime \prime \prime}\right)+O\left(\delta_{m}^{1-3 \lambda}\right) \\
& =O\left(\delta_{m}^{1-3 \lambda}\right) .
\end{aligned}
$$

Note that in the latter we exploited:

$\hat{t}_{i+1}-\hat{t}_{i}=\left(\hat{t}_{i+1}-\hat{t}_{i}\right)^{\lambda}\left(1+O\left(\delta_{m}^{2}\right)\right)$ yielding $\left(\hat{t}_{i+1}-\hat{t}_{i}\right)^{-2}=O\left(\delta_{m}^{-2 \lambda}\right)$. 
Indeed, recall that each regular curve $\gamma$ can be parameterized by arc-length (see [2]) giving $\|\dot{\gamma}(t)\|=1$ which upon differentiating $\|\dot{\gamma}(t)\|^{2}=\langle\dot{\gamma}(t) \mid \dot{\gamma}(t)\rangle=1$ yields $\langle\dot{\gamma}(t) \mid \ddot{\gamma}(t)\rangle=0$. The latter combined with

$\gamma\left(t_{i+1}\right)-\gamma\left(t_{i}\right)=\dot{\gamma}\left(t_{i}\right)\left(t_{i+1}-t_{i}\right)+(1 / 2) \ddot{\gamma}\left(t_{i}\right)\left(t_{i+1}-t_{i}\right)^{2}+O\left(\delta_{m}^{3}\right)$

and with (1.2) leads to: $\hat{t}_{i+1}-\hat{t}_{i}$

$$
=\left\|\gamma\left(t_{i+1}\right)-\gamma\left(t_{i}\right)\right\|^{\lambda}=\left\langle\gamma\left(t_{i+1}\right)-\gamma\left(t_{i}\right) \mid \gamma\left(t_{i+1}\right)-\gamma\left(t_{i}\right)\right\rangle^{\frac{\lambda}{2}}=\left(t_{i+1}-t_{i}\right)^{\lambda}\left(1+O\left(\delta_{m}^{2}\right)\right)^{\lambda} .
$$

Since $g(x)=(1+x)^{\lambda}=1+O(x)$ holds, for $x$ separated asymptotically from -1 , the substitution $x=O\left(\delta_{m}^{2}\right)$ in (4.21) justifies the first equation in (4.20). Furthermore as $\left(1+O\left(\delta_{m}\right)\right)^{2}=1+O\left(\delta_{m}\right)$ and since $h(x)=(1+x)^{-1}=$ $1+O(x)$, for $x$ separated asymptotically from -1 , the substitution $x=O\left(\delta_{m}\right)$ in the latter together with the first formula from (4.20) renders:

$\left(\hat{t}_{i+1}-\hat{t}_{i}\right)^{-2}=\left(t_{i+1}-t_{i}\right)^{-2 \lambda}\left(1+O\left(\delta_{m}^{2}\right)\right)^{-2}=\left(t_{i+1}-t_{i}\right)^{-2 \lambda}\left(1+O\left(\delta_{m}\right)\right)$.

Finally, the latter combined with $\beta \delta_{m} \leq t_{i+1}-t_{i}$ results in the second equation from (4.20) used ultimately to justify (4.19).

Newton interpolation formula (1.3) evaluated at any $\hat{t} \in \phi_{i}^{H}\left(I_{i}\right)$ for $\check{\gamma}_{i}^{H}(\hat{t})$ (see also (2.2)) yields all derivatives of $\check{\gamma}_{i}^{H}$ taken at $\hat{t}=\phi_{i}^{H}(t)$, with $t \in I_{i}$. Indeed the mean value theorem combined with (4.16) renders (here $\left.k=0,1\right)$ :

$\hat{t}-t_{i+k}=\frac{\phi_{i}^{H}(t)-\phi_{i}^{H}\left(t_{i+k}\right)}{t-t_{i+k}}\left(t-t_{i+k}\right)=\dot{\phi}_{i}^{H}\left(\xi_{i}\right)\left(t-t_{i+k}\right)=O\left(\dot{\phi}_{i}^{H}\right) \cdot O\left(\delta_{m}\right)=O\left(\delta_{m}^{\lambda}\right)$.

Thus by (4.17) and (4.19) we obtain:

$\hat{\gamma}_{i}^{H \prime}=O\left(\delta_{m}^{1-\lambda}\right)+O\left(\delta_{m}^{\lambda}\right) \cdot O\left(\delta_{m}^{1-2 \lambda}\right)+O\left(\delta_{m}^{2 \lambda}\right) \cdot O\left(\delta_{m}^{1-3 \lambda}\right)=O\left(\delta_{m}^{1-\lambda}\right)$,

$\hat{\gamma}_{i}^{H \prime \prime}=O\left(\delta_{m}^{1-2 \lambda}\right)+O\left(\delta_{m}^{\lambda}\right) \cdot O\left(\delta_{m}^{1-3 \lambda}\right)=O\left(\delta_{m}^{1-2 \lambda}\right), \quad$ and $\quad \hat{\gamma}_{i}^{H \prime \prime \prime}=O\left(\delta_{m}^{1-3 \lambda}\right)$

The asymptotics for (4.12) and (4.11) is next computed yielding the asymptotics for $f_{i}$ (see (4.1)).

\subsection{Step 4: Computing the Asymptotics for $f_{i}$}

Substituting (4.7), (4.8), (4.16) together with (4.22) and (4.23) into (4.12) renders the required asymptotics of $\rho_{i}\left[t_{i}, t_{i}, t_{i+1}, t_{i+1}, t\right]=O\left(\delta_{m}^{-3}\right)$,

for any $\lambda \in[0,1)$. Upon incorporating the latter into (4.11) we obtain (holding uniformly over $I_{i}$ ):

$\rho_{i}=O\left(\delta_{m}^{1}\right)+O\left(\delta_{m}^{4}\right) O\left(\delta_{m}^{-3}\right)=O\left(\delta_{m}\right)$.

Coupling (4.24) with (4.1) and (4.2) results in:

$f_{i}=\gamma-\check{\gamma}_{i}^{H} \circ \phi_{i}^{H}=O\left(\delta_{m}\right)+O\left(\delta_{m}\right)=O\left(\delta_{m}\right)$,

holding uniformly over each $I_{i}$. The proof is complete.

Recall that the uniform sampling $\mathcal{T}_{u}=\left\{t_{i}=(i T / m)\right\}_{i=0}^{m}$ (preserving more-or-less uniformity with $\beta=1$ ) yields accelerated orders in (4.7) and (4.8), by [12] equal to (see also [9]):

$\dot{\psi}_{i+k}=O\left(\delta_{m}^{\lambda-1}\right), \quad \ddot{\psi}_{i+k}=O\left(\delta_{m}^{\lambda}\right), \quad \dddot{\psi}_{i+k}=O\left(\delta_{m}^{\lambda-1}\right)$

and

$\check{\gamma}_{i+k}^{\prime}=O\left(\delta_{m}^{2-\lambda}\right), \quad \check{\gamma}_{i+k}^{\prime \prime}=O\left(\delta_{m}^{2-2 \lambda}\right), \quad \check{\gamma}_{i+k}^{\prime \prime \prime}=O\left(\delta_{m}^{3-3 \lambda}\right)$, 


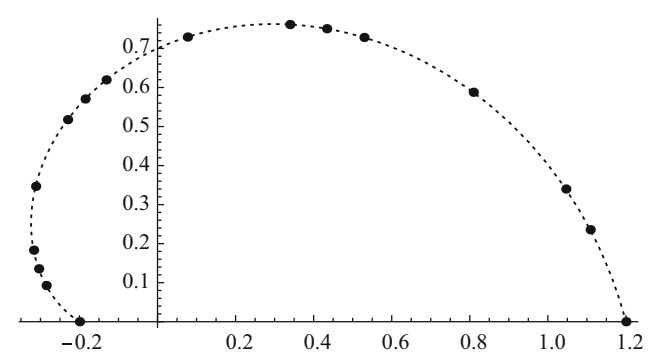

(a)

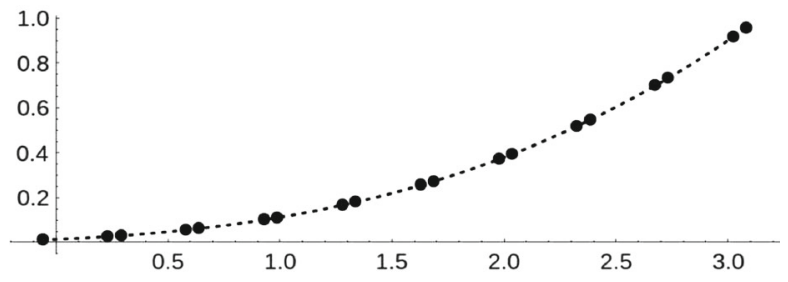

(b)

Fig. 1 a A spiral $\gamma_{s p}$ from (5.2). b A cubic $\gamma_{c}$ from (5.3)(i): sampled along a (5.1)(i), for $m=16$ or $\mathbf{b}(5.1)(i i)$, for $m=18$

for $k=0,1$. The latter combined with $\gamma-\check{\gamma}_{i} \circ \psi_{i}=O\left(\delta_{m}^{4}\right)$ (see $\left.[9,12]\right)$ when fed through to (4.1) renders accelerated quartic order in trajectory estimation. Indeed, upon repeating a similar argument as used to justify (3.2) one arrives at:

$$
\gamma-\check{\gamma}_{i}^{H} \circ \phi_{i}^{H}=O\left(\delta_{m}^{4}\right)
$$

holding uniformly over $[0, T]$. Note that the asymptotics in the latter (true for any $\lambda \in[0,1)$ and $\mathcal{T}_{u}$ uniform) coincides with (3.1) valid for $\lambda=1$ and arbitrary admissible sampling (1.1)—see Theorem 3.1.

In the next section we report on the numerical tests performed in Mathematica.

\section{Experiments}

In this section, the sharpness of Theorem 3.2 (see Remark 1) is numerically verified in affirmative. Additionally, the more-or-less uniformity condition assumed in Theorem 3.2 is also indicated as necessary via illustrative example. All numerical tests are carried out in Mathematica 10.0 — see also [23]. Three types of more-or-less uniform samplings $\mathcal{T}_{m o l}$ are used here to generate ordered sequences of reduced data $Q_{m}$ :

(i) $t_{i}=\left\{\begin{array}{ll}\frac{i}{m}+\frac{1}{2 m}, & \text { for } i=4 k+1, \\ \frac{i}{m}-\frac{1}{2 m}, & \text { for } i=4 k+3, \\ \frac{i}{m}, & \text { for } i=2 k,\end{array} \quad\right.$ (ii) $t_{i}=\frac{i}{m}+\frac{(-1)^{i+1}}{3 m}, \quad$ (iii) $t_{i}=\frac{i}{m}$.

For the first family $\mathcal{T}_{1}$ (see (5.1)(i)) $K_{l}=\frac{1}{2}, K_{u}=\frac{3}{2}$ and $\beta=\frac{1}{3}$-see Definition 1.2. The second family of samplings $\mathcal{T}_{2}$ (see $\left.(5.1)(i i)\right)$ yields $K_{l}=\frac{1}{3}, K_{u}=\frac{5}{3}$ and $\beta=\frac{1}{5}$. Lastly, the uniform sampling $\mathcal{T}_{u}$ (see (5.1)(iii)) renders $K_{l}=K_{u}=\beta=1$.

The four testing curves are $2 \mathrm{D}$ and $3 \mathrm{D}$ regular curves sampled along (5.1). The first three planar curves $\gamma_{s p}, \gamma_{c}, \gamma_{l}$ : $[0,1] \rightarrow \mathbb{E}^{2}$ represent either a a spiral:

$\gamma_{s p}(t)=((0.2+t) \cos (\pi(1-t)),(0.2+t) \sin (\pi(1-t)))$

or a cubic or a straight line:

(i) $\gamma_{c}(t)=\left(\pi t,(\pi t+1)^{3}(\pi+1)^{-3}\right)$ or $($ ii $) \gamma_{l}(t)=(t, 0)$.

The fourth $3 \mathrm{D}$ curve $\gamma_{q h}:[0,1] \rightarrow \mathbb{E}^{3}$ defines a quadratic elliptical helix by:

$\gamma_{q h}(t)=\left(2 \cos (2 \pi t), \sin (2 \pi t), 4 \pi^{2} t^{2}\right)$.

Figures 1 and 2 illustrate the respective trajectories of $\gamma_{s p}, \gamma_{c}$ and $\gamma_{q h}$ together with $Q_{18}=\left\{\gamma\left(t_{i}\right)\right\}_{i=0}^{18}$.

The $i$-th component of the error between continuous functions $\gamma$ and $\check{\gamma}^{H} \circ \phi^{H}$ over the compact set $\hat{I}_{i}$ is well-defined (according to Weierstrass extreme value theorem) and reads as:

$\mathcal{E}_{m}^{i}=\sup _{t \in\left[t_{i}, t_{i+1}\right]}\left\|\left(\check{\gamma}_{i}^{H} \circ \phi_{i}^{H}\right)(t)-\gamma(t)\right\|=\max _{t \in\left[t_{i}, t_{i+1}\right]}\left\|\left(\check{\gamma}_{i}^{H} \circ \phi_{i}^{H}\right)(t)-\gamma(t)\right\|$. 
Fig. 2 An elliptical helix $\gamma_{q h}$ from (5.4) sampled along (5.1)( $\mathrm{ii}$ ), for $m=18$

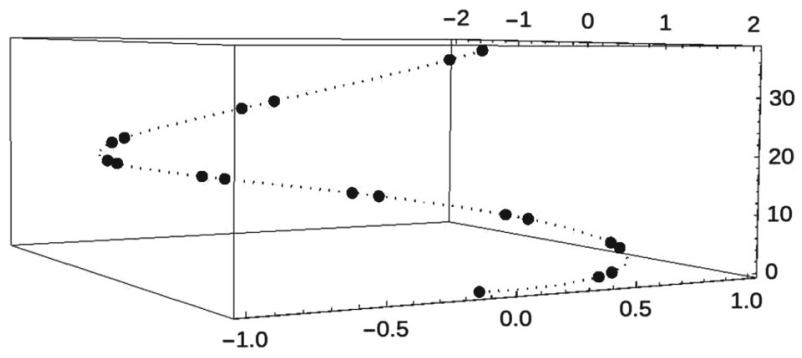

Table 1 Computed $\bar{\alpha}(\lambda) \approx \alpha(\lambda)$ for $\gamma_{s p}$ sampled as in $(5.1)$, for $m \in\{96, \ldots, 144\}$

\begin{tabular}{llllllll}
\hline$\lambda$ & 0.0 & 0.1 & 0.3 & 0.5 & 0.7 & 0.9 & 1.0 \\
\hline $\bar{\alpha}(\lambda)$ for $(5.1)(i)$ & 0.968 & 0.969 & 0.970 & 0.973 & 0.979 & 1.021 \\
$\bar{\alpha}(\lambda)$ for $(5.1)($ ii $)$ & 0.969 & 0.970 & 0.974 & 0.978 & 0.986 & 1.038 \\
$\alpha(\lambda)$ in Theorem 3.2 & 1.0 & 1.0 & 1.0 & 1.0 & 1.0 & 1.0 & 3.987 \\
$\bar{\alpha}(\lambda)$ for (5.1)(iii) & 3.995 & 3.993 & 3.989 & 3.939 & 3.934 & 3.933 \\
$\alpha(\lambda)$ in Theorem 3.2 & 4.0 & 4.0 & 4.0 & 4.0 & 4.0 & 4.0 & 3.933 \\
\hline
\end{tabular}

Table 2 Computed accelerated $\bar{\alpha}(\lambda)>\alpha(\lambda)$ for $\gamma_{s p}$ sampled along (5.1)(ii) for some $\lambda_{\varepsilon} \approx 1$ and for $m \in\{141, \ldots, 180\}$

\begin{tabular}{llllllllllll}
\hline$\lambda$ & 0.99300 & 0.99005 & 0.99700 & 0.99900 & 0.99950 & 0.99990 & 0.99993 & 0.99995 & 0.99997 & 0.99999 & 0.999993 \\
\hline $\bar{\alpha}(\lambda)$ & 1.6955 & 1.7882 & 1.8790 & 1.9569 & 1.9753 & 2.0528 & 2.112 & 2.2045 & 3.7210 & 3.9798 & 3.9917 \\
\hline
\end{tabular}

The total error over $[0, T]$ is taken as a maximal value $\mathcal{E}_{m}$ of the track-sum of $\mathcal{E}_{m}^{i}$ and is found here by using Mathematica optimization built-in functions: MaxValue and Max. From the set of the absolute errors $\left\{\mathcal{E}_{m}\right\}_{m=m_{\min }}^{m_{\max }}$ the numerical estimate $\bar{\alpha}(\lambda)$ of genuine convergence order $\alpha(\lambda)$ is subsequently computed by using linear regression. More specifically, Mathematica built-in function LinearModelFit returns the coefficient $\bar{\alpha}(\lambda)$ from the computed regression line $y(x)=\bar{\alpha}(\lambda) x+b$ applied to the collection of pairs of points $\left\{\left(\log (m),-\log \left(\mathcal{E}_{m}\right)\right)\right\}_{m_{\min }}^{m_{\max }}(\operatorname{see}[7])$.

Example 1 (i) The sharpness of Th 3.2 is tested first for the spiral $\gamma_{s p}$ defined in (5.2) sampled according to (5.1). To estimate $\alpha(\lambda)$ with $\bar{\alpha}(\lambda)$ a linear regression is applied with $96 \leq m \leq 144$. The generated numerical results are presented in Table 1. Visibly they are all consistent with the asymptotics established in Th 3.2. Note also that the accelerated quartic convergence orders established for either uniform sampling $\mathcal{T}_{u}$ or for $\lambda=1$ (see (3.1)) are also numerically confirmed as listed in Table 1 . However, a closer look for $\lambda_{\varepsilon}=1-\varepsilon \approx 1$ (with $\varepsilon>0$ very small) suggests for this particular case (i.e. for $\gamma_{s p}$ sampled along (5.1)(ii)) faster convergence rates $\bar{\alpha}\left(\lambda_{\varepsilon}\right)$ ranging continuously from 1 to 4 - see Table 2. Evidently, the results from Table 2 do not contradict (3.2). Indeed, the latter establishes merely the lower bounds on the respective $\alpha(\lambda) \geq 1$, for arbitrary $\lambda \in[0,1)$. Taking into account Remark 1, this example verifies numerically the sharpness of Theorem 3.2 at least for $\lambda \in[0,0.9]$. It also suggests that some continuous variations in accelerated $\alpha(\lambda)$ ranging from 1 to 4 are possible. Noticeably, any $\lambda \geq 0.9999995$ is rounded up by Mathematica to the value $\lambda=1.0$ and the resulting computed asymptotics stands for $\bar{\alpha}(1) \approx \alpha(1)=4$-see also Table 1 .

(ii) In an effort to verify the sharpness of (3.2) for $\lambda_{\varepsilon} \approx 1$ consider now a straight line $\gamma_{l}$ introduced in (5.3)(ii) and sampled according to (5.1)( $i$ i ). The corresponding numerical results are presented in Table 3 . In fact for $\lambda \in\{0,0.1,0.3,0.5,0.7,0.9\}$ numerical estimates $\bar{\alpha}(\lambda)=1.00$, for all $\lambda \in[0,1)$. Thus the sharpness of the asymptotics in Theorem 3.2 is confirmed numerically. Note also that for $\lambda=1$ both curves $\gamma_{l}$ and $\hat{\gamma}^{H} \circ \phi^{H}$ coincide yielding the machine error while estimating (5.5). 
Table 3 Computed $\bar{\alpha}(\lambda) \approx \alpha(\lambda)$ for $\gamma_{l}$ sampled along (5.1)(ii) for $\lambda_{\varepsilon} \approx 1$ and for $m \in\{141, \ldots, 180\}$

\begin{tabular}{llllllllllll}
\hline$\lambda$ & 0.99900 & 0.99930 & 0.99950 & 0.99970 & 0.99990 & 0.999930 & 0.99995 & 0.99997 & 0.99999 & 0.999995 & 0.999999 \\
\hline $\bar{\alpha}(\lambda)$ & 0.9999 & 0.9999 & 0.9999 & 0.9999 & 0.9999 & 0.9999 & 0.9999 & 0.9999 & 0.9999 & 0.9994 & 0.9977 \\
\hline
\end{tabular}

Table 4 Computed $\bar{\alpha}(\lambda) \approx \alpha(\lambda)$ for $\gamma_{c}$ sampled as in $(5.1)$, for $m \in\{96, \ldots, 144\}$

\begin{tabular}{llllllll}
\hline$\lambda$ & 0.0 & 0.1 & 0.3 & 0.5 & 0.7 & 0.9 & 1.0 \\
\hline $\bar{\alpha}(\lambda)$ for $(5.1)(i)$ & 0.981 & 0.981 & 0.982 & 0.983 & 0.987 & 1.002 & 3.997 \\
$\bar{\alpha}(\lambda)$ for (5.1)(ii) & 0.980 & 0.981 & 0.984 & 0.986 & 0.990 & 1.012 \\
$\alpha(\lambda)$ in Theorem 3.2 & 1.0 & 1.0 & 1.0 & 1.0 & 1.0 & 1.0 & 3.997 \\
$\bar{\alpha}(\lambda)$ for (5.1)(iii) & $*$ & 3.995 & 3.997 & 3.997 & 3.997 & 3.997 \\
$\alpha(\lambda)$ in Theorem 3.2 & 4.0 & 4.0 & 4.0 & 4.0 & 4.0 & 4.0 & 3.996 \\
\hline
\end{tabular}

*Machine error as $\hat{\gamma}^{H}=\gamma_{c}$

Table 5 Computed $\bar{\alpha}(\lambda) \approx \alpha(\lambda)$ for $\gamma_{q h}$ sampled as in (5.1), for $m \in\{110, \ldots, 162\}$

\begin{tabular}{llllllll}
\hline$\lambda$ & 0.0 & 0.1 & 0.3 & 0.5 & 0.7 & 0.9 & 1.0 \\
\hline $\bar{\alpha}(\lambda)$ for $(5.1)(i)$ & 0.969 & 0.961 & 0.963 & 0.966 & 0.973 & 1.007 & $4.052^{*}$ \\
$\bar{\alpha}(\lambda)$ for (5.1)(ii) & 0.965 & 0.967 & 0.972 & 0.976 & 0.983 & 1.022 & $3.805 * *$ \\
$\alpha(\lambda)$ in Theorem 3.2 & 1.0 & 1.0 & 1.0 & 1.0 & 1.0 & 1.0 & 4.0 \\
$\bar{\alpha}(\lambda)$ for (5.1)(iii) & 4.000 & 3.895 & 3.872 & 3.864 & 3.866 & 3.875 & 3.882 \\
$\alpha(\lambda)$ in Theorem 3.2 & 4.0 & 4.0 & 4.0 & 4.0 & 4.0 & 4.0 & 4.0 \\
\hline
\end{tabular}

$* 69 \leq m \leq 120 ; * * 160 \leq m \leq 200$

Example 2 In the next step, the sharpness of Theorem 3.2 is tested for the cubic $\gamma_{c}$ defined in (5.3)(i) and sampled along (5.1). To compute numerically $\alpha(\lambda)$ with $\bar{\alpha}(\lambda)$ a linear regression is used for $m$ varying within the set $96 \leq m \leq 144$. Again the generated results listed in Table 4 are consistent with the asymptotics proved in Theorem 3.2. Moreover, faster convergence orders established for either uniform sampling $\mathcal{T}_{u}$ or for $\lambda=1$ (see (3.1)) are also numerically confirmed in Table 4 .

Example 3 The next example reports on numerical experiments testing the sharpness of Theorem 3.2 for the quadratic elliptical helix $\gamma_{q h}$ (see (5.4)) sampled according to (5.1). To examine numerically $\alpha(\lambda)$ with $\bar{\alpha}(\lambda)$ a linear regression is used for $m$ varying within the set $110 \leq m \leq 162$. Since (3.2) has asymptotic character (i.e. it holds for sufficiently large $m$ ) for some $\lambda \in[0,1]$ other intervals of integers are used. The results presented in Table 5 confirm the asymptotics from (3.2). In addition, fast quartic convergence orders prevailing for either uniform sampling $\mathcal{T}_{u}$ or for $\lambda=1$ (see (3.1)) are numerically confirmed as listed in Table 5.

The last example shows that more-or-less uniformity assumption cannot be dropped in Theorem 3.2.

Example 4 Consider $\gamma_{s p}$ from (5.2) sampled according to the non-more-or-less uniform sampling:

$t_{i}=\left\{\begin{array}{lll}\frac{i}{m}, & \text { for } i & \text { even, } \\ \frac{i-1}{m}+\frac{1}{m^{2}}, & \text { for } i & \text { odd. }\end{array}\right.$

Such sampling is not permissible in Theorem 3.2. On one hand, the numerical tests reported in Table 6 for $\lambda \in[0,1)$ indicate either divergence in $\gamma_{s p}-\check{\gamma}^{H} \circ \phi^{H}$ whenever $\bar{\alpha}(\lambda)$ is negative or otherwise strong deceleration effects with $0<\bar{\alpha}(\lambda)<1$. The corresponding absolute errors $\mathcal{E}_{144}$ (see (5.5)) between $\gamma_{s p}-\check{\gamma}^{H} \circ \phi^{H}$ confirm independently 
Table 6 Computed $\bar{\alpha}(\lambda)$ for $\gamma_{s p}$ sampled as in (5.6) for $m \in\{96, \ldots, 144\}$

\begin{tabular}{llllllll}
\hline$\lambda$ & 0.0 & 0.1 & 0.3 & 0.5 & 0.7 & 0.9 & 1.0 \\
\hline $\bar{\alpha}(\lambda)$ for $(5.2)$ & -2.068 & -1.621 & -1.436 & -0.598 & 0.068 & 0.536 & 3.980 \\
$\mathcal{E}_{144}$ & 69713.7 & 5810.44 & 171.753 & 12.243 & 0.514 & 0.013 & $6.99 \cdot 10^{-8}$ \\
\hline
\end{tabular}

such tendency. On the other hand, since $\lambda=1$ assumes a general class of admissible samplings (1.1) (see Theorem 3.1 ) and since (4) is still admissible, Table 6 renders a sharp estimate $\bar{\alpha}(1)=3.980 \approx \alpha(1)=4$ with marginal absolute error $\mathcal{E}_{144}=6.9910^{-8}$.

\section{Conclusions}

This work's main contribution extends the claim of Theorem 3.1 (established for $\lambda=1$-see [7] or [8]) to all remaining $\lambda \in[0,1)$ controlling exponential parameterization (1.2). The latter coupled with modified Hermite interpolant $\hat{\gamma}^{H}$ (see Sect. 2) is discussed here in the context of fitting reduced data $Q_{m}$ in arbitrary Euclidean space $\mathbb{E}^{n}$. Conditions enforcing at least linear convergence order $\alpha(\lambda)=1$ (for $\lambda \in[0,1)$ to estimate $\gamma$ by $\hat{\gamma}^{H} \circ \phi^{H}$ are formulated in Theorem 3.2 (see Sect. 3). The underlying asymptotics (3.2) for $\gamma-\hat{\gamma} \circ \phi^{H}$ is ultimately proved in Sect. 4. Finally, Sect. 5 reports on numerical tests (conducted in Mathematica) confirming the sharpness of the asymptotics determined by (3.1) and (3.2). The necessity of more-or-less uniformity stipulated by Theorem 3.2 is also substantiated numerically.

Future work may e.g. focus on proving analytically the sharpness of the above asymptotics or on enforcing its acceleration within certain subfamilies of more-or-less uniform samplings. The other possible extension of this work is to examine the impact of both $\delta_{m}$ and $Q_{m}^{P}$ representing a small perturbation of data points $Q_{m}$ on the difference between $\gamma$ and $\hat{\gamma}$ fitting $Q_{m}^{P}$. Lastly establishing sufficient conditions ensuring that $\phi^{H}$ defines a reparameterization of $[0, T] \rightarrow[0, \hat{T}]$ is also in priority demands. Related problem was addressed in the context of $C^{0}$ piecewisequadratic Lagrange interpolation in [9-11]. The reparameterization problem is vital for curve modelling (e.g. for a trajectory planning) and length estimation $d(\gamma)$ based on either sparse or dense reduced data. For example, in trajectory planning some applications like inspecting electrical poles by drones may require extra looping. For such a task, no reparameterization condition imposed on $\phi$ is needed. In contrast, the length estimation $d(\gamma)$ by $d(\hat{\gamma})$ relies on the existence of genuine re-reparameterization $\phi:[0, T] \rightarrow[0, \hat{T}]$, at best preserving fast convergence rates (see e.g. $[5,16])$.

Related research on fitting reduced data can also be found e.g. in $[3,13,14,17,21,22]$. A similar effect of the left-hand side discontinuity in convergence rate jumping from 1 to 3 (or from 1 to 4 ) at $\lambda=1$ occurs for $C^{0}$ piecewise-quadratic (piecewise-cubic) Lagrange interpolation based on exponential parameterization and $Q_{m}-$ see $[9,10,12,20]$. Some accelerations in trajectory estimation can be achieved for the so-called $\varepsilon$-uniformly sampled reduced data $Q_{m}$ (see e.g. [7,10,12]).

In particular, the complete cubic spline $\hat{\gamma}^{C S} \in C^{2}$ (see e.g. [1]) incorporating $Q_{m}$, both velocities $\dot{\gamma}\left(t_{0}\right)=$ $v_{0}, \dot{\gamma}\left(t_{m}\right)=v_{m}$ and cumulative chord parameterization (i.e.for $\lambda=1$ in (1.2)) is discussed in [5]. The latter proves the quartic convergence rate $\alpha(1)=4$ in estimating $\gamma$ by $\hat{\gamma}^{C S}$. However, the remaining cases of $\lambda \in[0,1)$ in (1.2) are not addressed in [5]. On the other hand, if both initial and terminal velocities are not supplied, the modified complete spline $\hat{\gamma}_{M}^{C S} \in C^{2}$ defined exclusively by $Q_{m}$ and (1.2) is numerically tested in [13]. Here, the ending velocities $v_{0}$ and $v_{n}$ are similarly approximated as outlined in Sect. 2. The latter results in numerically estimated quartic (linear) convergence order in approximating $\gamma$ by $\hat{\gamma}_{M}^{C S}$, for $\lambda=1$ (or for $\lambda \in\left[0,1\right.$ ). Alternatively, for $v_{0}$ and $v_{n}$ unavailable, the natural spline $\hat{\gamma}^{N S} \in C^{2}$ (see e.g. [1]) based on $Q_{m},(1.2)$ and ad hock adopted constraints $\hat{\gamma}^{N S^{\prime \prime}}\left(\hat{t}_{0}\right)=\hat{\gamma}^{N S^{\prime \prime}}\left(\hat{t}_{m}\right)=\overrightarrow{0}$ can be applied. As numerically verified in [14], the decelerated quadratic (linear) convergence order in approximating $\gamma$ by $\hat{\gamma}^{N S}$ prevails, for $\lambda=1$ (for $\lambda \in[0,1$ ). In contrast to Theorem 3.2, no analytic proof is so-far given to justify the underlying asymptotics to approximate $\gamma$ by either $\hat{\gamma}_{M}^{C S}$ or by $\hat{\gamma}^{N S}$. 
Fitting reduced data forms an important problem in computer vision and graphics, engineering, microbiology, physics and other applications like medical image processing (e.g. for area and boundary computation or trajectory and length estimation)—see e.g. [4,6,15,16,18].

Open Access This article is distributed under the terms of the Creative Commons Attribution 4.0 International License (http:// creativecommons.org/licenses/by/4.0/), which permits unrestricted use, distribution, and reproduction in any medium, provided you give appropriate credit to the original author(s) and the source, provide a link to the Creative Commons license, and indicate if changes were made.

\section{References}

1. de Boor, C.: A Practical Guide to Spline. Springer, Berlin (1985)

2. do Carmo, M.P.: Differential Geometry of Curves and Surfaces. Prentice-Hall, Engelwood Cliffs (1976)

3. Epstein, M.P.: On the influence of parameterization in parametric interpolation. SIAM J. Numer. Anal. 13, 261-268 (1976)

4. Farin, G.: Curves and Surfaces for Computer Aided Geometric Design. Academic Press, San Diego (1993)

5. Floater, M.S.: Chordal cubic spline interpolation is fourth order accurate. IMA J. Numer. Anal. 26(1), 25-33 (2005)

6. Janik, M., Kozera, R., Kozioł, P.: Reduced data for curve modeling - applications in graphics, computer vision and physics. Adv. Sci. Technol. 7(18), 28-35 (2013)

7. Kozera, R.: Curve modeling via interpolation based on multidimensional reduced data. Stud. Inf. 25(4B-61), 1-140 (2004)

8. Kozera, R., Noakes, L.: $C^{1}$ interpolation with cumulative chord cubics. Fundam. Inf. 61(3-4), 285-301 (2004)

9. Kozera, R., Noakes, L.: Piecewise-quadratics and exponential parameterization for reduced data. Appl. Math. Comput. 221, 620-638 (2013)

10. Kozera, R., Noakes, L.: Piecewise-quadratics and $\varepsilon$-uniformly sampled reduced data. Appl. Math. Inf. Sci. 10(1), 33-48 (2016)

11. Kozera, R., Noakes, L.: Piecewise-quadratics and reparameterizations for interpolating reduced data. In: Proceedings of Computer Algebra in Scientific Computing, LNCS 9301, pp. 260-274. Springer, Berlin (2015)

12. Kozera, R.L., Wilkołazka, M.: Convergence order in trajectory estimation by piecewise cubics and exponential parameterization (in press)

13. Kozera, R., Noakes, L., Wilkołazka, M.: A modified complete spline interpolation and exponential parameterization. Lecture Notes in Computer Science 9339, pp. 98-110. Springer, Berlin (2015)

14. Kozera, R., Noakes, L., Wilkołazka, M.: A natural spline interpolation and exponential parameterization. In: Proceedings of American Institute of Physics 1738, pp. 180003-1-183003-4. AIP Publishing (2016)

15. Kozera, R., Wilkołazka, M.: A natural spline interpolation and exponential parameterization for length estimation of curves. In: Proceedings of American Institute of Physics 1863, pp. 400010-1-400010-4. AIP Publishing (2017)

16. Kozera, R., Noakes, L., Szmielew, P.: Convergence orders in length estimation with exponential parameterization and $\varepsilon$-uniformly sampled reduced data. Appl. Math. Inf. Sci. 10(1), 107-115 (2016)

17. Kuznetsov, E.B., Yakimovich, A.Y.: The best parameterization for parametric interpolation. J. Appl. Math. 191, 239-245 (2006)

18. Kvasov, B.I.: Methods of Shape-Preserving Spline Approximation. World Scientific Publishing Company, Singapore (2000)

19. Lee, E.T.Y.: Choosing nodes in parametric curve interpolation. Comput.-Aided Des. 21(6), 363-370 (1989)

20. Noakes, L., Kozera, R.: Cumulative chords piecewise-quadratics and piecewise-cubics. In: Klette, R., Kozera, R., Noakes, L., Weickert, J. (eds.) Geometric Properties from Incomplete Data, Computational Imaging and Vision, vol. 31, pp. 59-75. Kluver Academic Publisher, Dordrecht (2006)

21. Piegl, L., Tiller, W.: The NURBS Book. Springer, Berlin (1997)

22. Rababah, A.: High order approximation methods for curves. Comput. Aided Geom. Des. 12, 89-102 (1995)

23. Wolfram, S.: The Mathematica Book, 5th edn. Wolfram Media, Champaign (2003) 